УДК 519.1

\author{
I. V. Protasov, K. D. Protasova
}

\title{
METRICALLY RAMSEY ULTRAFILTERS
}

\begin{abstract}
I. V. Protasov, K. D. Protasova. Metrically Ramsey ultrafilters, Mat. Stud. 49 (2018), 115-121.
Given a metric space $(X, d)$, we say that a mapping $\chi:[X]^{2} \longrightarrow\{0,1\}$ is an isometric coloring if $d(x, y)=d(z, t)$ implies $\chi(\{x, y\})=\chi(\{z, t\})$. A free ultrafilter $\mathcal{U}$ on an infinite metric space $(X, d)$ is called metrically Ramsey if, for every isometric coloring $\chi$ of $[X]^{2}$, there is a member $U \in \mathcal{U}$ such that the set $[U]^{2}$ is $\chi$-monochrome. We prove that each infinite ultrametric space $(X, d)$ has a countable subset $Y$ such that each free ultrafilter $\mathcal{U}$ on $X$ satisfying $Y \in \mathcal{U}$ is metrically Ramsey. On the other hand, it is an open question whether every metrically Ramsey ultrafilter on the natural numbers $\mathbb{N}$ with the metric $|x-y|$ is a Ramsey ultrafilter. We prove that every metrically Ramsey ultrafilter $\mathcal{U}$ on $\mathbb{N}$ has a member with no arithmetic progression of length 2 , and if $\mathcal{U}$ has a thin member then there is a mapping $f: \mathbb{N} \longrightarrow \omega$ such that $f(\mathcal{U})$ is a Ramsey ultrafilter.
\end{abstract}

For any finite coloring of the set $[\mathbb{N}]^{2}$ of edges of the complete graph on the set $\mathbb{N}$ of natural numbers, there exists an infinite subset $A \subseteq \mathbb{N}$ such that the set $[A]^{2}$ is monochrome.

This elegant statement is a graph version of Ramsey theorem, one of the milestone of Ramsey Theory. For history (with exposition of the original paper of Frank Ramsey) and foundations of this branch of Combinatorics, see [2]. Some isometric versions of Ramsey theorem can be found in [7], [8].

We recall that a family $\mathfrak{F}$ of subsets of a set $X$ is a filter if $\varnothing \notin \mathfrak{F}, \mathfrak{F}$ is closed under finite intersections and if $A \in \mathfrak{F}$ and $A \subseteq B$ then $B \in \mathfrak{F}$. The family of all filters on $X$ is ordered by inclusion $\subseteq$, and a filter maximal in this ordering is called an ultrafilter. A filter $\mathcal{U}$ is an ultrafilter if and only if, for any finite partition $\mathcal{P}$ of $X$, there exists $A \in \mathcal{P}$ such that $A \in \mathcal{U}$. An ultrafilter $\mathcal{U}$ is called free if $\bigcap \mathcal{U}=\varnothing$.

Is there a free ultrafilter $\mathcal{U}$ of $\mathbb{N}$ such that, for each coloring $\chi:[\mathbb{N}]^{2} \longrightarrow\{0,1\}$, there exists $A \in \mathcal{U}$ such that $[A]^{2}$ is $\chi$-monochrome? These ultrafilters are called Ramsey ultrafilters. The question can not be answered in the system ZFC of axioms of Set Theory without additional set-theoretical assumptions. If we accept the Continuum Hypothesis, the answer is positive. On the other hand, there are models of ZFC without Ramsey ultrafilters.

Let $X$ be an infinite set and let $\mathfrak{F}$ be some family of $\{0,1\}$-colorings of the set $[X]^{2}$ of all two-element subsets of $X$. We say that a free ultrafilter $\mathcal{U}$ on $X$ is Ramsey with respect to $\mathfrak{F}$ if, for any coloring $\chi \in \mathfrak{F}$, there exists $U \in \mathcal{U}$ such that $[U]^{2}$ is $\chi$-monochrome. In the case in which $\mathfrak{F}$ is the family of all $\{0,1\}$-colorings of $[X]^{2}$, we get above definition of Ramsey ultrafilters. It is well-known that $\mathcal{U}$ is a Ramsey ultrafilter if and only if $\mathcal{U}$ is selective, i.e. for every partition $\mathcal{P}$ of $X$ either $P \in \mathcal{U}$ for some $P \in \mathcal{P}$ or there exists $U \in \mathcal{U}$ such that $|U \cap P| \leq 1$ for each $P \in \mathcal{P}$.

2010 Mathematics Subject Classification:05D10.

Keywords: selective ultrafilter; metrically Ramsey ultrafilter; ultrametric space.

doi:10.15330/ms.49.2.115-121

(C) I. V. Protasov, K. D. Protasova, 2018 
Given a metric space $(X, d)$, we say that a mapping $\chi:[X]^{2} \longrightarrow\{0,1\}$ is an isometric coloring if $d(x, y)=d(z, t)$ implies $\chi(\{x, y\})=\chi(\{z, t\})$. We note that every isometric coloring $\chi$ is uniquely defined by some mapping $f: d(X, X) \backslash\{0\} \longrightarrow\{0,1\}$. Indeed, we take an arbitrary $r \in d(X, X) \backslash\{0\}$, choose $\{x, y\} \in[X]^{2}$ such that $d(x, y)=r$ and put $f(r)=\chi(\{x, y\})$. On the other hand, for $f: d(X, X) \backslash\{0\} \rightarrow\{0,1\}$, we define $\chi$ by $\chi(\{x, y\})=f(d(x, y))$.

We say that a free ultrafilter on an infinite metric space $(X, d)$ is metrically Ramsey if $\mathcal{U}$ is Ramsey with respect to all isometric colorings of $[X]^{2}$.

Let $G$ be a group and let $X$ be a $G$-space with the action $(G, X) \longrightarrow X,(g, x) \longmapsto g x$. A coloring $\chi:[X]^{2} \longrightarrow\{0,1\}$ is called $G$-invariant if $\chi(\{x, y\})=\chi(\{g x, g y\})$ for all $\{x, y\} \in$ $[X]^{2}$ and $g \in G$. A free ultrafilter $\mathcal{U}$ of $X$ is called $G$-Ramsey if $\mathcal{U}$ is Ramsey with respect to the family of all $G$-invariant colorings of $[X]^{2}$.

We consider the special case: $X$ is a metric space and $G$ is a group of isometries of $X$. Clearly, every isometric coloring of $[X]^{2}$ is $G$-invariant. If $G$ is metrically 2-transitive (if $d(x, y)=d(z, t)$ then there is $g \in G$ such that $g\{x, y\}=\{z, t\})$ then every $G$-invariant coloring of $[X]^{2}$ is an isometric coloring.

We take the group $\mathbb{Z}$ of integers, put $X=\mathbb{Z}$ and consider the action $\mathbb{Z}$ on $X$ by $(g, x)=$ $g+x$. Is every $\mathbb{Z}$-Ramsey ultrafilter selective? This question appeared in [5] and, to our knowledge, remains open. We endow $\mathbb{Z}$ with the metric $d(x, y)=|x-y|$. By above paragraph an ultrafilter $\mathcal{U}$ on $\mathbb{Z}$ is $\mathbb{Z}$-Ramsey if and only if $\mathcal{U}$ is metrically Ramsey. Is every metrically Ramsey ultrafilter on $\mathbb{Z}$ selective? This is an equivalent form of the above question. The case of $\mathbb{Z}$ evidently equivalent to the case of $\mathbb{N}$.

Surprisingly or not, the case of ultrametric spaces is cardinally different and much more easy to explore. We recall that a metric $d$ is an ultrametric if $d(x, y) \leq \max \{d(x, z), d(z, y)\}$ for all $x, y, z \in X$. We prove that every infinite ultrametric space $X$ has a countable subset $Y$ such that any ultrafilter $\mathcal{U}$ on $X$ satisfying $Y \in \mathcal{U}$ is metrically Ramsey.

1. Equidistance subsets. We say that a subset $Y$ of a metric space $(X, d)$ is an equidistance subset if there is $r \in \mathbb{R}^{+}$such that $d(x, y)=r$ for all distinct $x, y \in Y$. If $Y$ is an equidistance subset of $(X, d)$ then every free ultrafilter $\mathcal{U}$ on $X$ such that $Y \in \mathcal{U}$ is metrically Ramsey.

Proposition 1. Every infinite metric space with finite scale $d(X, X), d(X, X)=\{d(x, y)$ : $x, y \in X\}$ has a countable equidistance subset.

Proof. We define a coloring $\chi:[X]^{2} \longrightarrow d(X, X)$ by $\chi(\{x, y\})=d(x, y)$ and apply the classical Ramsey theorem [2, p.16].

For an ultrametric space $(X, d)$ and $r \in d(X, X)$, we use the equivalence $\sim_{r}$ defined by $x \sim_{r} y$ if and only if $d(x, y) \leq r$. Then $X$ is partitioned into classes of $r$-equivalence $X=\bigcup_{\alpha<\lambda} X_{\alpha}$. If $x, y \in X_{\alpha}$ then $d(x, y) \leq r$. If $x \in X_{\alpha}, y \in X_{\beta}$ and $\alpha \neq \beta$ then $d(x, y)>r$.

Proposition 2. Let $(X, \alpha)$ be an infinite ultrametric space with finite scale $d(X, X)$. If $|X|$ is regular then $X$ has an equidistance subset $Y$ of cardinality $|Y|=|X|$. If $|X|$ is singular then, for every cardinal $\kappa<|X|$, there is an equidistance subset of cardinality $\kappa$.

Proof. Let $d(X, X)=\left\{0, r_{1}, \ldots, r_{n}\right\}, 0<r_{1}<\ldots<r_{n}$. We proceed by induction on $n$. For $n=1$, the statement is evident: $Y=X$.

To make the inductive step from $n$ to $n+1$, we partition $X$ into classes of $r_{n}$-equivalence $X=\bigcup_{\alpha<\lambda} X_{\alpha}$. If $\lambda=|X|$ then we pick one element $y_{\alpha} \in X_{\alpha}$ and put $Y=\left\{y_{\alpha}: \alpha<|X|\right\}$, 
so $d(x, y)=r_{n+1}$ for all distinct $x, y \in Y$. Assume that $\lambda<|X|$. If $|X|$ is regular, we take $\alpha$ so that $\left|X_{\alpha}\right|=X$ and apply the inductive assumption to $X_{\alpha}$. If $|X|$ is singular then we take $X_{\alpha}$ such that $\left|X_{\alpha}\right|>\kappa$ and use the inductive assumption.

Remark 1. If in Proposition $1.2|X|$ is singular, we cannot state that there is an equidistance subset $Y$ of cardinality $X$. We take an arbitrary singular cardinal $\kappa$, put $X=\kappa$ and partition $X=\bigcup_{\alpha<\lambda} X_{\alpha}$ so that $\lambda<\kappa$ and $\left|X_{\alpha}\right|<\kappa$ for each $\alpha<\kappa$. We define an ultrametric $d$ on $X$ by $d(x, x)=0, d(x, y)=1$ if $x, y \in X_{\alpha}, x \neq y$ and $d(x, y)=2$ if $x \in X_{\alpha}, y \in X_{\beta}, \alpha \neq \beta$. If $Y$ is an equidistance subset of $(X, \alpha)$ then either $Y \subseteq X_{\alpha}$ for some $\alpha<\lambda$, or $\left|Y \cap X_{\alpha}\right| \leq 1$ for each $\alpha<\lambda$. Hence, $|Y|<\kappa$.

Proposition 3. For every infinite cardinal $\kappa$, there exists a metric space $(X, d)$ such that $|X|=2^{\kappa}, d(X, X)=\{0,1,2\}$ and every equidistance subset $Y$ of $(X, d)$ is of cardinality $|Y| \leq \kappa$.

Proof. We put $X=2^{\kappa}$ and apply [4, Theorem 6.2] to define a coloring $\chi:[X]^{2} \longrightarrow\{1,2\}$ with no monochrome $[Z]^{2}$ for $|Z|>\kappa$. Then we define a metric $d$ on $X$ by $d(x, x)=0$ and $d(x, y)=\chi(\{x, y\})$ for all distinct $x, y \in X$.

Proposition 4. Let $(X, d)$ be a metric space with infinite scale $d(X, X),|d(X, X)|=\kappa$. If $|X| \geq\left(2^{\kappa}\right)^{+}$then there is an equidistance subset $Y$ of $(X, d)$ such that $|Y|=\kappa^{+}$.

Proof. We define a coloring $\chi:[X]^{2} \longrightarrow d(X, X)$ by $\chi(\{x, y\})=d(x, y)$ and apply the Erdös-Rado theorem [4, Theorem 6.4].

Proposition 5. For every infinite metric space $(X, d)$, there exists an injective sequence $\left(x_{n}\right)_{n \in \omega}$ in $X$ such that one of the following conditions is satisfied:

(i) the sequence $\left(d\left(x_{0}, x_{n}\right)\right)_{n \in \omega}$ is increasing;

(ii) the sequence $\left(d\left(x_{0}, x_{n}\right)\right)_{n \in \omega}$ is decreasing;

(iii) for every $n \in \omega$ and all $i, j, i>n, j>n$, we have $d\left(x_{n}, x_{i}\right)=d\left(x_{n}, x_{j}\right)$.

Proof. We assume that there exists $x_{0} \in X$ such that the set $d\left(x_{0}, X\right)$ is infinite, $d\left(x_{0}, X\right)=$ $\left\{d\left(x_{0}, x\right): x \in X\right\}$. We choose a countable subset $Y$ of $X$ such that $x_{0} \notin Y$ and $d\left(x_{0}, y\right) \neq$ $d\left(x_{0}, z\right)$ for all distinct $y, z \in Y$. The set $d\left(x_{0}, Y\right)$ contains either increasing or decreasing sequence $\left(r_{n+1}\right)_{n \in \omega}$. For each $n \in \omega$, we choose $x_{n+1}$ such that $d\left(x_{0}, x_{n+1}\right)=r_{n}$. Then the sequence $\left(d\left(x_{0}, x_{n}\right)\right)_{n \in \omega}$ satisfies either $(i)$ or $(i i)$.

In the alternative case, the set $d(x, X)$ is finite for each $x \in X$. We fix $x_{0} \in X$ and choose a countable subset $X_{1}$ such that $\left|d\left(x_{0}, X_{1}\right)\right|=1$. We pick $x_{1} \in X_{1}$ and choose a countable subset $X_{2} \subseteq X_{1}$ such that $\left|d\left(x_{1}, X_{2}\right)\right|=1$ and so on. After $\omega$ steps, we get the sequence $\left(x_{n}\right)_{n \in \omega}$ satisfying $(i i i)$.

Proposition 6. Let $(X, d)$ be an infinite metric space and let $\left\{X_{n}: n \in \omega\right\}$ be a family of non-empty pairwise disjoint subsets of $X$ such that $d\left(X_{i}, X_{j}\right) \bigcap d\left(X_{n}, X_{n}\right)=\varnothing$ for all $n$ and distinct $i, j$. Let $\mathcal{U}$ be a metrically Ramsey ultrafilter on $X$ such that $\bigcup_{n<\omega} X_{n} \in \mathcal{U}$ and $X_{n} \notin \mathcal{U}$ for each $n<\omega$. Then the following statements hold:

(i) there exists $U \in \mathcal{U}$ such that $\left|U \cap X_{n}\right| \leq 1$ for each $n<\omega$;

(ii) if $d\left(X_{i}, X_{j}\right) \bigcap d\left(X_{k}, X_{l}\right)=\varnothing$ for all distinct $\{i, j\},\{k, l\} \in[\omega]^{2}$ then there is a mapping $\varphi: X \longrightarrow \omega$ such that the ultrafilter $\varphi(\mathcal{U})$ is selective; 
(iii) if for each $n<\omega$ there exists $m>n,\left|X_{m}\right|>n$, then there exists an ultrafilter $\mathcal{V}$ on $X$ such that $\bigcup_{n<\omega} X_{n} \in \mathcal{V}$ and $\mathcal{V}$ is not metrically Ramsey.

Proof. ( $i)$ By the assumption, the sets $A=\bigcup_{n<\omega} d\left(X_{n}, X_{n}\right)$ and $B=\bigcup_{i \neq j} d\left(X_{i}, X_{j}\right)$ are disjoint. We take an arbitrary mapping $f: \mathbb{R}^{+} \longrightarrow\{0,1\}$ such that $\left.f\right|_{A} \equiv 0,\left.f\right|_{B} \equiv 1$, and consider the isometric coloring $\chi$ of $[X]^{2}$ defined by $f$. Since $\mathcal{U}$ is metrically Ramsey, there is $U \in \mathcal{U}$ such that $[U]^{2}$ is $\chi$-monochrome. Clearly, $\left|U \cap X_{n}\right| \leq 1$ for each $n<\omega$.

(ii) We define $\varphi$ by the rule: if $x \in X_{i}$ then $\varphi(x)=i$, if $x \in X \backslash \bigcup_{n<\omega} X_{n}$, then $\varphi(x)=0$. We take an arbitrary coloring $\chi^{\prime}:[\omega]^{2} \longrightarrow\{0,1\}$ and define a coloring $\chi:\left[\bigcup_{n<\omega} X_{n}\right]^{2} \longrightarrow\{0,1\}$ as follows. If $x \in X_{i}, y \in X_{j}, i \neq j$ then $\chi(\{x, y\})=\chi^{\prime}(\{x, y\})$. If $x, y \in X_{n}$ then $\chi(\{x, y\})=$ 0 . By the assumption, the coloring $\chi$ is isometric. We choose $U \in \mathcal{U}$ such that $U \subseteq \bigcup_{n<\omega} X_{n}$ and $[U]^{2}$ is $\chi$-monochrome and $\left|U \bigcap X_{n}\right| \leq 1$ for each $n<\omega$. Then $\varphi(U) \in \varphi(\mathcal{U})$ and $[\varphi(U)]$ is $\chi^{\prime}$-monochrome, so $\varphi(\mathcal{U})$ is a Ramsey ultrafilter.

(iii) We consider the family of all filters $\mathfrak{F}$ on $X$ such that $\bigcup_{n<\omega} X_{n} \in \mathfrak{F}$ and, for every $n \in \omega$ and $F \in \mathfrak{F}$, there exists $m \in \omega$ such that $\left|F \cap X_{m}\right|>n$. By the Zorn Lemma, this family has maximal by inclusion element $\mathcal{V}$. It is easy to verify that $\mathcal{V}$ is ultrafilter. By $(i), \mathcal{V}$ is not metrically Ramsey.

\section{The ultrametric case.}

Proposition 7. For every infinite ultrametric space $(X, d)$, there exists a countable subset $Y$ of $X$ such that every free ultrafilter $\mathcal{U}$ on $X$ satisfying $Y \in \mathcal{U}$ is metrically Ramsey.

Proof. We choose the sequence $\left(x_{n}\right)_{n \in \omega}$ given by Proposition 1.5, put $Y=\left\{x_{n}: n \in \omega\right\}$, fix an arbitrary mapping $f: \mathbb{R}^{+} \longrightarrow\{0,1\}$ and take an arbitrary free ultrafilter $\mathcal{U}$ satisfying $Y \in \mathcal{U}$

We assume that either $(i)$ or $(i i)$ of Proposition 1.5 holds for $\left(x_{n}\right)_{n \in \omega}$. We define a mapping $h: Y \longrightarrow \mathbb{R}^{+}$by $h\left(x_{n}\right)=d\left(x_{0}, x_{n}\right)$ and choose $k \in\{0,1\}$ such that $(f h)^{-1}(k) \in \mathcal{U}$. Since $d$ is an ultrametric, in the case $(i)$ we have $d\left(x_{i}, x_{n}\right)=d\left(x_{0}, x_{n}\right)$ for all $i<n$, and in the case (ii) we have $d\left(x_{i}, x_{n}\right)=d\left(x_{0}, x_{i}\right)$ for all $i<n$. In both cases, if $\left\{x_{i}, x_{n}\right\} \in\left[(f h)^{-1}(k)\right]^{2}$ then $f\left(d\left(x_{i}, x_{n}\right)\right)=k$.

If $\left(x_{n}\right)_{n \in \omega}$ satisfies $(i i i)$ of Proposition 1.5 then we define a mapping $h: Y \longrightarrow \mathbb{R}^{+}$by $h\left(x_{n}\right)=d\left(x_{n}, x_{i}\right), i>n$ and repeat the above arguments.

Proposition 8. For a free ultrafilter $\mathcal{U}$ on an infinite set $X$, the following statements are equivalent:

(i) $\mathcal{U}$ is selective;

(ii) $\mathcal{U}$ is metrically Ramsey for each ultrametric $d$ on $X$ such that $d(X, X)=\{0,1,2\}$.

Proof. The implication $(i) \Longrightarrow(i i)$ is evident. To show $(i i) \Longrightarrow(i)$, we assume that $\mathcal{U}$ is not selective and choose a partition $\mathcal{P}$ of $X$ such that $P \notin \mathcal{U}$ for each $P \in \mathcal{P}$, and for every $U \in \mathcal{U}$, there is $P \in \mathcal{P}$ such that $|P \cap U|>1$. We define an ultrametric $d$ on $X$ by $d(x, x)=0, d(x, y)=1$ if $x \neq y, x, y \in P$ for some $P \in \mathcal{P}$, and $d(x, y)=2$ if $x, y$ belong to different cells of the partition $\mathcal{P}$. We define a coloring $\chi:[X]^{2} \longrightarrow\{1,2\}$ by $\chi(\{x, y\})$ $=d(x, y)$. Then the set $[U]^{2}$ is not $\chi$-monochrome for each $U \in \mathcal{U}$ so $\mathcal{U}$ is not metrically Ramsey and $(i i) \Longrightarrow(i)$.

Proposition 9. Let $(X, d)$ be an infinite ultrametric space with finite scale $d(X, X)=$ $\left\{0, r_{1}, \ldots, r_{n}\right\}, 0<r_{1}<\ldots<r_{n}$. Then the following statements are equivalent: 
(i) every free ultrafilter on $(X, d)$ is metrically Ramsey;

(ii) for every $i \in\{1, \ldots, n\}$, the partition $\mathcal{P}_{i}$ of $X$ into classes of $r_{i}$-equivalence has only finite number of infinite classes and there is $m \in \omega$ such that $|C|<m$ for each finite class $C$ from $\mathcal{P}_{i}$.

Proof. $(i) \Longrightarrow(i i)$. If $\mathcal{P}_{i}$ has infinitely many infinite classes or the set $\{|C|: C$ is a finite class from $\left.\mathcal{P}_{i}\right\}$ is infinite we apply Proposition 1.6(iii) to get a free ultrafilter $\mathcal{V}$ on $X$ which is not metrically Ramsey.

$(i i) \Longrightarrow(i)$. We proceed on induction by $n$. For $n=1$, the statement is evident.

To make the inductive step from $n$ to $n+1$, we take an arbitrary free ultrafilter $\mathcal{U}$ on $X$ and we consider the partition $\mathcal{P}_{n+1}$. Let $X_{1}, \ldots, X_{m}$ be the set of all infinite classes from $\mathcal{P}_{n+1}$. If $X_{1} \cup \ldots \cup X_{m} \in \mathcal{U}$ then we take $X_{i} \in \mathcal{U}$ and apply the inductive assumption. If $X \backslash\left(X_{1} \bigcup \ldots \cup X_{m}\right) \in \mathcal{U}$ then we choose $U \in \mathcal{U}$ such that $U \subseteq X \backslash\left(X_{1} \cup \ldots \cup X_{m}\right)$ and $|U \cap C| \leq 1$ for each finite class $C \in \mathcal{P}_{n+1}$. Then $U$ is an equidistance set so $\mathcal{U}$ is metrically Ramsey.

\section{The case of $\mathbb{N}$.}

Proposition 10. Let $\mathcal{U}$ be a metrically Ramsey ultrafilter on $\mathbb{N}$ and let $f: \mathbb{N} \longrightarrow \mathbb{N}$ be a mapping such that $f(x)>x$ for each $x \in \mathbb{N}$. Then there exists a member $U \in \mathcal{U}$ having no subsets of the form $\{a, a+x, a+f(x)\}$. In particular ( for $f(x)=2 x$ ), some member of $\mathcal{U}$ has no arithmetic progressions of length 2.

Proof. We consider a directed graph $\Gamma_{f}$ with the set of vertices $\mathbb{N}$ and the set of edges $\{(x, f(x)): x \in \mathbb{N}\}$. Since $f(x)>x, \Gamma_{f}$ is the disjoint union of directed trees $T$ such that each vertex of $T$ has at most one input edge. Using this observation, it is easy to partition $\mathbb{N}=A_{1} \cup A_{2}$ so that $f\left(A_{1}\right) \subseteq A_{2}, \quad f\left(A_{2}\right) \subseteq A_{1}$.

The partition $\mathbb{N}=A_{1} \bigcup A_{2}$ defines an isometric coloring $\chi:[\mathbb{N}]^{2} \longrightarrow\{1,2\}$ by $\chi(\{x, y\})=$ $i$ if and only if $d(x, y) \in A_{i}$. We take a subset $U \in \mathcal{U}$ such that the set $[U]^{2}$ is $\chi$-monochrome and assume that $\{a, a+x, a+f(x)\} \subset U$ for some $a, x \in \mathbb{N}$. We note that $d(a, a+x)=x$, $d(a, a+f(x))=f(x)$, but $x$ and $f(x)$ belong to disjoint subsets $A_{1}, A_{2}$, so $\chi(\{a, a+x\}) \neq$ $\chi(\{a, a+f(x)\})$ and we get a contradiction with the choice of $U$.

Let $\mathcal{U}$ be metrically Ramsey ultrafilter on $\mathbb{N}$. Assume that there is $U \in \mathcal{U}$ such that $d(x, y) \neq d(z, t)$ for all distinct $\{x, y\},\{z, t\} \in[U]^{2}$. Then every $\{0,1\}$-coloring of $[U]^{2}$ can be extended to some isometric coloring of $[\mathbb{N}]^{2}$. Hence, $\mathcal{U}$ is a Ramsey ultrafilter.

We say that a subset $T=\left\{t_{n}: t_{n}<t_{n+1}, n<\omega\right\}$ of $\mathbb{N}$ is thin if $\left(t_{n+1}-t_{n}\right) \longrightarrow \infty$ as $n \longrightarrow \infty$.

Proposition 11. If a metrically Ramsey ultrafilter $\mathcal{U}$ on $\mathbb{N}$ has a thin subset $T \in \mathcal{U}$ then there exists a mapping $\varphi: \mathbb{N} \longrightarrow \omega$ such that the ultrafilter $\varphi(\mathcal{U})$ is selective and $\varphi$ is finite-to-one on some member $U \in \mathcal{U}$.

Proof. Let $T=\left\{t_{n}: t_{n}<t_{n+1}, n \in \omega\right\}$. Assume that we have chosen two sequences $\left(a_{n}\right)_{n \in \omega}$, $\left(b_{n}\right)_{n \in \omega}$ in $T$ such that

(1) $a_{n}<b_{n}<a_{n+1}<b_{n+1}$ for each $n \in \omega$;

(2) $d\left(\left[a_{n}, b_{n}\right) \cap T,\left[a_{n}, b_{n}\right) \cap T\right) \cap d\left(\left[a_{i}, b_{i}\right) \cap T,\left[a_{j}, b_{j}\right) \cap T\right)=\varnothing$ for all $n$ and distinct $i, j$;

(3) $d\left(\left[a_{i}, b_{i}\right) \cap T,\left[a_{j}, b_{j}\right) \cap T\right) \bigcap d\left(\left[a_{k}, b_{k}\right) \cap T,\left[a_{l}, b_{l}\right) \cap T\right)=\varnothing$ for all distinct $\{i, j\}, \quad\{k, l\} \in$ 
$[\omega]^{2}$

(4) $d\left(\left[b_{n}, a_{n+1}\right) \cap T,\left[b_{n}, a_{n+1}\right) \cap T\right) \bigcap d\left(\left[b_{i}, a_{i+1}\right) \cap T,\left[b_{j}, a_{j+l}\right) \cap T\right)=\varnothing$ for all $n$ and distinct $i, j$;

(5) $d\left(\left[b_{i}, a_{i+1}\right) \cap T,\left[b_{j}, a_{j+1}\right) \cap T\right) \cap d\left(\left[b_{k}, a_{k+1}\right) \cap T,\left[b_{l}, a_{l+l}\right) \cap T\right)=\varnothing$ for all distinct $\{i, j\}$, $\{k, l\} \in[\omega]^{2}$.

We put $A=\bigcup_{n \in \omega}\left(\left[a_{n}, b_{n}\right) \cap T\right), B=\bigcup_{n \in \omega}\left(\left[b_{n}, a_{n+1}\right) \cap T\right)$ and note that $A$ and $B$ with the corresponding partitions satisfy Proposition $1.6(i i)$. Since either $A \in \mathcal{U}$ or $B \in \mathcal{U}$, Proposition $1.6($ ii $)$ gives the mapping $\varphi: \mathbb{N} \longrightarrow \omega$ such that $\varphi(\mathcal{U})$ is selective. By the construction of $\varphi, \varphi$ is finite-to-one on $A$ or $B$ respectively.

It remains to construct $\left(a_{n}\right)_{n \in \omega}$ and $\left(b_{n}\right)_{n \in \omega}$. We put $a_{0}=t_{0}, b_{0}=t_{1}$ and assume that we have chosen $a_{0}, b_{0}, \ldots, a_{n}, b_{n}$. Since $T$ is thin, we can choose $a_{n+1} \in T$ so that $a_{n+1}>2 b_{n}$ and $\left|t-t^{\prime}\right|>2 a_{n}$ for all distinct $t, t^{\prime} \in T \backslash\left[1, a_{n+1}\right)$. Then we choose $b_{n+1} \in T$ so that $b_{n+1}>2 a_{n+1}$ and $\left|t-t^{\prime}\right|>2 b_{n}$ for all distinct $t, t^{\prime} \in T \backslash\left[1, b_{n+1}\right)$. After $\omega$ steps, we get the desired $\left(a_{n}\right)_{n \in \omega},\left(b_{n}\right)_{n \in \omega}$.

4. Comments and open questions. 1. In connection with Proposition 3.1, we mention [5, Corollary 2]: every metrically Ramsey ultrafilter on $\mathbb{N}$ has a member $U$ with no subsets of the form $\{x, y, x+y\}, x \neq y$.

In connection with Proposition 3.2, we ask

Question 1. Let $\mathcal{U}$ be a metrically Ramsey ultrafilter on $\mathbb{N}$. Does there exist a thin subset $U \in \mathcal{U}$ ?

Question 2. Assume that a metrically Ramsey ultrafilter $\mathcal{U}$ on $\mathbb{N}$ has a thin member. Is $\mathcal{U}$ selective?

2. Let $G$ be an Abelian group. A coloring $\chi:[G]^{2} \longrightarrow\{0,1\}$ is called a PS-coloring if, for $\{x, y\},\{z, t\} \in[G]^{2}, x+y=z+t$ implies $\chi(\{x, y\})=\chi(\{z, t\})$. A free ultrafilter $\mathcal{U}$ on $G$ is called a PS-ultrafilter if $\mathcal{U}$ is Ramsey with respect to all PS-colorings of $[G]^{2}$. The PS-ultrafilters were introduced and studied in [6], for an exposition of [6] see [1, Chapter 10].

If $G$ has a finite set $B(G)=\{g \in G: 2 g=0\}$ of elements of order 2 then every PSultrafilter on $G$ is selective. If $B(G)$ is infinite then, under Martin's Axiom, there is a nonselective PS-ultrafilter on $G$. If there exists PS-ultrafilter on some countable group $G$ then there is a $P$-point in $\omega^{*}$.

Now we consider the countable Boolean group $\mathbb{B}, B(\mathbb{B})=\mathbb{B}$. We note that a coloring $\chi:[\mathbb{B}]^{2} \longrightarrow\{0,1\}$ is a PS-coloring if and only if $\chi$ is $\mathbb{B}$-invariant. Thus, a free ultrafilter $\mathcal{U}$ on $\mathbb{B}$ is a PS-ultrafilter if and only if $\mathcal{U}$ is $\mathbb{B}$-Ramsey. By the above paragraph, in the models of ZFC with no P-points in $\omega^{*}$, there are no $\mathbb{B}$-Ramsey ultrafilters. However, every strongly summable ultrafilter on $\mathbb{B}$ is a $P S$-ultrafilter. For strongly summable ultrafilters on Abelian groups, see [3].

On the other hand, $\mathbb{B}$ is the direct sum $\oplus_{n<\omega}\{0,1\}_{n}$ of $\omega$ copies of $\mathbb{Z}_{2}=\{0,1\}$, and has the natural structure of the ultrametric space $(\mathbb{B}, d)$, where $d\left(\left(x_{n}\right)_{n \in \omega},\left(y_{n}\right)_{n \in \omega}\right)=\min \left\{m: x_{n}=\right.$ $\left.y_{n}, n \geq m\right\}$. By Proposition 2.1, there are plenty metrically Ramsey ultrafilters on $\mathbb{B}$ in ZFC. Applying Proposition 1.6 (iii), we can find ultrafilters on $\mathbb{B}$ which are not metrically Ramsey.

3. By [4, Theorem 6.2], there is a coloring $\chi:[\mathbb{R}]^{2} \longrightarrow\{0,1\}$ such that if $X \subset \mathbb{R}$ and $[X]^{2}$ is $\chi$-monochrome then $|X| \leq \omega$.

We endow $\mathbb{R}$ with the natural metric $d(x, y)=|x-y|$ and we ask 
Question 4.3. Does there exist an isometric coloring $\chi:[\mathbb{R}]^{2} \longrightarrow\{0,1\}$ such that if $[X]^{2}$ is monochrome then $|X| \leq \omega$ ?

We endow the Cantor cube $\{0,1\}^{\omega}$ with the standard metric and we ask

Question 4.4. Does there exist an isometric coloring $\chi:\left[\{0,1\}^{\omega}\right]^{2} \rightarrow\{0,1\}$ such that if $[X]^{2}$ is monochrome then $|X| \leq \omega$ ?

\section{REFERENCES}

1. M. Filali, I. Protasov, Ultrafilters and topologies on groups, Math. Stud. Monogr. Ser., V.13, VNTL, Lviv, 2010.

2. R. Graham, B. Rotschild, J. Spencer, Ramsey Theory, Willey, New York, 1980.

3. N. Hindman, I. Protasov, D. Strauss, Strongly summable ultrafilters on Abelian groups, Mat. Stud., 10 (1998), №2, 121-132.

4. K. Kunen, Combinatories, in: Handbook in Mathematical Logic, V.90 (Studies in Logic on Foundations of Mathematics, J. Barwise (editor)), Elsevier, 1982.

5. O. Petrenko, I. Protasov, Selective and Ramsey ultrafilters on G-spaces, Notre Dame J. Formal Logic, 58 (2017), 453-459.

6. I. Protasov, Ultrafilters and partitions of Abelian groups, Ucr. Mat. Zh., 53 (2001), 85-93; translation in Ukr. Math. J. 53 (2001), 99-107.

7. I. Protasov, Isometric versions of Ramsey theorem, EUREKA, 65 (2017), 25-27.

8. I. Protasov, On colorings and isometries, Proc. Intern. Geometry Center, 10, (2017), №2, 1-7; translation in Ukr. Math. J. 53 (2001), 99-107.

Faculty of Computer Science and Cybernetics

Kyiv University, Kyiv, Ukraine

i.v.protasov@gmail.com

ksuha@freenet.com.ua 